\title{
THE DEGREE OF A SEVERI VARIETY
}

\author{
ZIV RAN
}

Consider the curves of some fixed degree $d$ in the complex projective plane $\mathbf{P}^{2}$. These are parametrized by a projective space $P_{d}$, projectivization of the vector space of homogeneous polynomials of degree $d$ in three variables. By a Severi variety we mean either the (locally closed) algebraic subset of $P_{d}$ corresponding to curves with a fixed number of nodes (i.e., points where the curve has two transverse smooth branches) and no other singularities, or the subset of the latter corresponding to irreducible curves. These varieties have received considerable attention since they were introduced by Enriques [1] and Severi [7]. For an expository account, see [2] or [5]. For instance the famous Severi problem, recently solved by Harris [3], is to prove the former authors' claim that a Severi variety parametrizing irreducible curves is itself irreducible.

With the Severi problem now out of the way, the time seems ripe to begin asking some finer questions about the Severi varieties. One such question is: what is the degree, in $P_{d}$, of a Severi variety? The purpose of this announcement is to give a recursive procedure for answering this question. This procedure involves, even in its statement, a generalization of the Severi varieties to varieties of nodal curves on the blowup $\tilde{\mathbf{P}}^{2}$ of $\mathbf{P}^{2}$ at a point. We give a formula (see Theorem 2) for the degree of a generalized Severi variety for curves of given "type" $(d, e), 0 \leq e \leq d-2$, and given number of nodes in terms of degrees of similar varieties for curves of various types $\left(d^{\prime}, e^{\prime}\right)$ where $e^{\prime}>e$ or $d^{\prime}<d$. Since the degree of a generalized Severi variety for curves of type $(d, d-1)$ is easily computed, this gives an effective procedure for computing the degree of any generalized Severi variety. Among the generalized Severi varieties, the "ordinary" ones are those for type $(d, 0)$. Thus even if one only cares about the latter, he is led by the above procedure to the former.

1. Setup. Let $b: \tilde{\mathbf{P}}^{2} \rightarrow \mathbf{P}^{2}$ denote the blowing up of a point, with exceptional divisor $E$, and for integers $d \geq e \geq 0$ let $L_{d, e}$ denote the line bundle $b^{*} O(d)(-e E)$ on $\tilde{\mathbf{P}}^{2}$. For

$$
-d+1 \leq g \leq \frac{(d-1)(d-2)}{2}-\frac{e(e-1)}{2}
$$

(respectively,

$$
\left.0 \leq g \leq \frac{(d-1)(d-2)}{2}-\frac{e(e-1)}{2}\right)
$$

denote by $V^{d, g, e}$ (resp. $\left.V(d, g, e)\right)$ the (locally closed) subset of the complete linear system $\left|L_{d, e}\right|$ consisting of (possibly reducible) (resp. irreducible) nodal

Received by the editors October 9, 1986 and, in revised form, February 10, 1987. $14 \mathrm{D} 20$.

1980 Mathematics Subject Classification (1985 Revision). Primary 14H10; Secondary

Research partially supported by the NSF. 
curves of (geometric) genus $g$ (or equivalently, with $\delta$ nodes,

$$
\left.\delta=\frac{(d-1)(d-2)}{2}-\frac{e(e-1)}{2}-g\right)
$$

note that the genus of a reducible curve is defined by the formula $g\left(\bigcup_{1}^{k} C_{i}\right)=$ $\sum g\left(C_{i}\right)-k+1$. It is easily seen (cf. [4]) that $V^{d, g, e}$ and $V(d, g, e)$ are both nonempty, nonsingular and purely of dimension $3 d+g-e-1$. The following result can be proved with a slight modification in the methods of [4].

THEOREM 1. $V(d, g, e)$ is irreducible.

Note that $V^{d, g, e}$ consists of $V(d, g, e)$ plus other components which are the various products (in general, mixed cartesian-symmetric) of other $V\left(d^{\prime}, g^{\prime}, e^{\prime}\right)$ 's corresponding to the ways a curve may split up.

We now fix some notation and terminology concerning partitions. For us, a partition $\pi$ is given by a sequence of nonnegative integers $\left(w_{1}, \ldots, w_{n}\right)$, determined up to affixing a tail of zeros. A divisor on a smooth curve is of type $\pi$ if it has the form $\sum_{i=1}^{n} \sum_{j=1}^{w_{i}} i P_{i j}$, for some distinct points $P_{i j}$. The weight of $\pi$ is $|\pi|=\sum i w_{i}$ and the length is $\langle\pi\rangle=\sum w_{i}$; also put $[\pi]=|\pi|-\langle\pi\rangle$, $\langle\pi|=w_{1}$. The notions of (vector) sum $\pi+\pi^{\prime}$, domination $\pi \leq \pi^{\prime}$ and difference $\pi^{\prime}-\pi$ (if $\pi \leq \pi^{\prime}$ ) are defined in the obvious way. If $\pi=\left(w_{1}, \ldots, w_{n}\right) \leq$ $\pi^{\prime}=\left(w_{1}^{\prime}, \ldots, w_{n}^{\prime}\right)$, put $\left(\begin{array}{c}\pi^{\prime} \\ \pi\end{array}\right)=\prod\left(\begin{array}{c}w_{i}^{\prime} \\ w_{i}\end{array}\right)$. Define a function $F$ on partitions by $F(\pi)=\prod i^{w_{i}}$. Finally put $N(\pi)=\left(\begin{array}{c}\langle\pi\rangle \\ w_{1}, \ldots, w_{n}\end{array}\right) \prod i^{w_{i}}$, and note that this is the degree of the variety of divisors of type $\pi$ on $\mathbf{P}^{1}$.

2. Formula. For $(d, g, e)$ as in $\S 1$, we denote by $\Delta(d, g, e)\left(\right.$ resp. $\left.\Delta^{i}(d, g, e)\right)$ the degree of $V^{d, g, e}$ (resp. $V(d, g, e)$ ) in the projective space $\left|L_{d, e}\right|$; otherwise set $\Delta(d, g, e)$ or $\Delta^{i}(d, g, e)$ to equal 0 . More generally, denote by $\Delta\left(d, g, e, \pi_{v}, \pi_{f}, \pi^{v}, \pi^{f}\right)$ the degree of the locus of curves $C$ in $V^{d, g, e}$ whose intersection with some fixed general line $L$ (resp. the exceptional divisor $E$ ) consists of some fixed divisor of type $\pi_{f}$ (resp. $\pi^{f}$ ) plus a variable divisor of type $\pi_{v}$ (resp. $\pi^{v}$ ), all supported at smooth points of $C$. Here $\left|\pi_{v}+\pi_{f}\right|=d$, $\left|\pi^{v}+\pi^{f}\right|=e$. Finally, define $\Delta^{u}\left(d, g, e, \pi_{v}, \ldots, \pi^{f}\right)$ analogously to $\Delta$ except that the curves are assumed not to contain any ruling. The following theorem yields a recursive procedure for computing all these functions.

THEOREM 2. (i) We have $\Delta\left(d, \ldots, \pi^{f}\right)=\Delta^{u}\left(d, \ldots, \pi^{f}\right)=0$ unless

$$
-d+1 \leq g \leq \frac{(d-1)(d-2)}{2}-\frac{e(e-1)}{2}
$$

and $\left[\pi_{v}+\pi^{v}\right]+\left|\pi_{f}+\pi^{f}\right| \leq 3 d+g-e-1$. Also $\Delta^{u}\left(d, g, e, \ldots, \pi^{f}\right)=0$ for $g \leq e-d$, and $\Delta^{i}(d, g, e)=0$ for $g<0$.

(ii) We have

$$
\begin{aligned}
& \Delta\left(d,-h, d-1, \pi_{v}, \ldots, \pi^{f}\right) \\
& \quad=\sum\left(\begin{array}{c}
\left\langle\pi_{f}\right| \\
r_{f}
\end{array}\right)\left(\begin{array}{c}
\left\langle\pi^{f}\right| \\
r^{f}
\end{array}\right)\left(\begin{array}{c}
2 d-h-\left[\pi_{v}+\pi^{v}\right]-\left|\pi_{f}+\pi^{f}\right| \\
h-r_{f}-r^{f}
\end{array}\right) \\
& \quad \cdot N\left(\pi_{v}-\left(h-r_{f}\right)\right) N\left(\pi^{v}-\left(h-r^{f}\right)\right) ;
\end{aligned}
$$


here and elsewhere, a summand involving a difference $\pi^{\prime}-\pi$ where $\pi \not \pi^{\prime}$ is taken to be zero.

$$
\Delta^{u}(d,-h, d-1, \ldots)= \begin{cases}0, & h>0 \\ \Delta(d, 0, d-1, \ldots), & h=0\end{cases}
$$

(iii) We have

$$
\begin{aligned}
& \Delta^{u}\left(d, g, e, \pi_{v}, \pi^{v}, \pi_{f}, \pi^{f}\right) \\
& \quad=\sum\left(\begin{array}{c}
\left\langle\pi_{f}\right| \\
r_{f}
\end{array}\right)\left(\begin{array}{c}
\left\langle\pi^{f}\right| \\
r^{f}
\end{array}\right)\left(\begin{array}{c}
3 d+g-e-1-\left[\pi_{v}+\pi^{v}\right]-\left|\pi_{f}+\pi^{f}\right| \\
r-r_{f}-r^{f}
\end{array}\right) \\
& \quad \cdot \Delta^{u}\left(d-r, g+r, e-r, \pi_{v}-\left(r-r_{f}\right), \pi_{f}-\left(r_{f}\right), \pi^{v}-\left(r-r^{f}\right), \pi^{f}-\left(r^{f}\right)\right) .
\end{aligned}
$$

(iv) We have

$$
\Delta(d, g, e)=\sum \frac{1}{m_{1} ! \cdots m_{s} !}\left(\begin{array}{c}
n \\
n_{1} \cdots n_{r}
\end{array}\right) \Delta^{i}\left(d_{1}, g_{1}, e_{1}\right) \cdots \Delta^{i}\left(d_{1}, g_{1}, e_{r}\right),
$$

the sum being over all unordered collections $\left(d_{i}, g_{i}, e_{i}\right), i=1, \ldots, r$ with $\sum d_{i}=d, \sum e_{i}=e, \sum g_{i}-r+1=g, 3 d_{i}+g_{i}-e_{i}-1=n_{i}, n=\sum n_{i}$, and where $m_{j}$ denotes the number of times some distinct triple $\left(d_{j}, g_{j}, e_{j}\right)$ appears in the above collection.

(v) Suppose $e \leq d-2$ and $g \geq 3-2 d+2 e$, and fix any pair of integers $n_{1} \geq d+1, n_{2} \geq e+1$ with $n_{1}+n_{2}=3 d+g-e-1$. Then we have

$$
\begin{aligned}
& \Delta\left(d, g, e, \pi_{v}, \pi_{f}, \pi^{v}, \pi^{f}\right) \\
& =\sum F\left(\pi_{1}+\pi_{2}\right)\left(\begin{array}{c}
\pi^{f} \\
\pi_{0}^{f}
\end{array}\right)\left(\begin{array}{c}
\pi^{v} \\
\pi_{0}^{v}
\end{array}\right)\left(\begin{array}{c}
\pi_{v} \\
\pi_{v}^{0}
\end{array}\right)\left(\begin{array}{c}
\pi_{f} \\
\pi_{f}^{0}
\end{array}\right) \\
& \cdot \Delta\left(d-\left|\pi_{v}^{0}+\pi_{f}^{0}\right|, g^{\prime}+\left|\pi_{v}^{0}+\pi_{f}^{0}\right|, e^{\prime}-\pi_{v}^{0}+\pi_{f}^{0},\right. \\
& \left.\quad \pi_{v}-\pi_{v}^{0}, \pi_{f}-\pi_{f}^{0}, \pi_{1}+\pi_{0}^{v}, \pi_{2}+\pi_{0}^{f}\right) \\
& \cdot \Delta\left(e^{\prime}-\left|\pi_{0}^{v}+\pi_{0}^{f}\right|, g^{\prime \prime}+\left|\pi_{0}^{v}+\pi_{0}^{f}\right|, e-\left|\pi_{0}^{v}+\pi_{0}^{f}\right|,\right. \\
& \left.\pi_{2}+\pi_{v}^{0}, \pi_{1}+\pi_{f}^{0}, \pi^{v}-\pi_{v}-\pi_{0}^{v}, \pi^{f}-\pi_{0}^{f}\right),
\end{aligned}
$$

the summation being subject to the restrictions $e<e^{\prime}<d, g^{\prime}+g^{\prime \prime}=g-e^{\prime}+$ $\pi_{1}+\pi_{2}+1,3 d+g^{\prime}-e^{\prime}-1 \geq n_{1}+\left[\pi_{1}+\pi_{0}^{v}\right]+\left|\pi_{2}+\pi_{0}^{f}\right|, 3 e^{\prime}+g^{\prime \prime}-e-1 \geq$ $n_{2}+\left|\pi_{1}+\pi_{f}^{0}\right|+\left[\pi_{2}+\pi_{0}^{v}\right]$ and $\pi_{1}+\pi_{2}+\pi_{v}^{0}+\pi_{f}^{0}+\pi_{0}^{v}+\pi_{0}^{f}=e^{\prime}$.

REMARKS. 1. The hypothesis $g \geq 3-2 d+2 e$ guarantees the existence of at least one pair $\left(n_{1}, n_{2}\right)$ as in (v).

2. It is easy to translate the theorem into an algorithm for computing $\Delta$, $\Delta^{u}$, and $\Delta^{i}$ : namely, first, (i), (ii) and (iii) immediately yield their values for $e=d-1$. Next, suppose we know their values for all $\left(d_{0}, g_{0}, e_{0}\right)$ with $d_{0}<d$ or $e_{0}>e$, for some $(d, g, e)$ with $e<d-1$. If $g \leq 2-2 d+2 e$ (which is $\leq e-d$ ), then a combination of (i) and (iii) immediately yields the values of $\Delta$ and $\Delta^{u}$ and then (iv) yields $\Delta^{i}$. If $g \geq 3-2 d+2 e$, then (v) first yields the value of $\Delta$, then an application of (iii) and (iv) yield the values of $\Delta^{u}$ and $\Delta^{i}$.

3. It is also straightforward to translate the theorem into a closed-form formula for any of the $\Delta$-functions. However, such a formula would be quite complicated. From the computational viewpoint, the recursive approach seems 
more practical. Of course, the possibility still remains that a simple, closedform formula exists.

4. Example: Let $f(d)=\Delta\left(d, \frac{d(d-3)}{2}, 0\right)$. Taking $n_{1}=d+1$, the above yields

$$
\begin{aligned}
f(d) & =\Delta(d, 0, d-1) f(d-1)+\Delta(d,-1, d-1) \Delta\left(d-1, \frac{(d-2)(d-3)}{2}, 0\right) \\
+2 \Delta & (d, 0, d-1,(0),(0),(0),(0,1)) \Delta\left(d-1, \frac{(d-2)(d-3)}{2},(0,1),(0),(0),(0)\right) \\
& =f(d-1)+2 d-1+4(d-2) \\
& =f(d-1)+6 d-9 .
\end{aligned}
$$

Since $f(1)=0$, we recover the well-known result $f(d)=3(d-1)^{2}$.

5. Analogous formulas may be given for other related loci of curves, such as those with $n$ nodes and 1 cusp (resp. 1 tacnode, resp. 1 triple point). Details will be given elsewhere.

3. Sketch of proof. Parts (i)-(iv) of Theorem 2 are trivial, and have been included only for the sake of completeness. The main point is part $(\mathrm{v})$. Its proof is based on the method used in [4] to prove the irreducibility of $V(d, g, e)$. Consider a 1-parameter degeneration with general fibre $\tilde{\mathbf{P}}^{2}$ and special fibre $\tilde{\mathbf{P}}_{1}^{2} \cup \tilde{\mathbf{P}}_{2}^{2}$ with $\tilde{\mathbf{P}}_{1}^{2} \cap \tilde{\mathbf{P}}_{2}^{2}=E$ the exceptional divisor on $\tilde{\mathbf{P}}_{1}^{2}$ and a line-section on $\tilde{\mathbf{P}}_{2}^{2}$, and the limit of $V^{d, g, e}$ in this family, say in the sense of the Hilbert scheme (the method of generalized linear systems [6] provides another, somewhat clearer way of taking this limit, but this is not essential). The methods of [4] show that this limit consists of the various loci of curves of the form $C_{1} \cup C_{2}$ with $C_{1} \in V^{d, g^{\prime}, e^{\prime}}$ on $\tilde{\mathbf{P}}_{1}^{2}, C_{1} \in V^{e^{\prime}, g^{\prime \prime}, e}$ on $\tilde{\mathbf{P}}_{2}^{2}$ with $e \leq e^{\prime} \leq d, g^{\prime}+g^{\prime \prime}=g-e^{\prime}+t+1$, and $C_{1} \cap E=C_{2} \cap E$ a divisor of type $\pi$ smooth on $C_{1}$ and $C_{2}$, with $g^{\prime}+g^{\prime \prime}=g-e^{\prime}+[\pi]+1$, this locus appearing with multiplicity $F(\pi)$. For simplicity, assume $\pi=(0, t)$. The contribution of the latter locus to $\Delta(d, g, e)$ is then computed by identifying the set of $t$-tuples on $E$ with $\mathbf{P}^{t}$ and using the Kunneth decomposition of the diagonal class on $\mathbf{P}^{t} \times \mathbf{P}^{t}$. More general cases are done similarly.

\section{REFERENCES}

1. F. Enriques, Sui moduli d'una classe di superficie e sul teorema d'esistenza per funzioni algebriche di due variabili, Atti Accad. Sci. Torino 47 (1912).

2. W. Fulton, On nodal curves, Algebraic Geometry: open problems, Lecture Notes in Math., vol. 997, Springer-Verlag, New York, 1983, pp. 146-155.

3. J. Harris, On the Severi problem, Invent. Math. 84 (1986), 445-461.

4. Z. Ran, On nodal plane curves, Invent. Math. 86 (1986), 529-534.

5. _ The Severi problem: a post-mortem (?), Mathematical Aspects of String Theory, S. T. Yau, ed. (to appear).

6. __ Degeneration of linear systems (preprint).

7. F. Severi, Vorlesungen über Algebraische Geometrie, Teubner, Leipzig, 1921.

Department of MATHEMATICS AND COMPUTER SCIENCE, UNiversity of CALIFORNiA, RIVERSIDE, CALIFORNIA 92521

Current address: Institut Mittag-Leffler, Auravägen 17, S18262 Djursholm, Sweden 